\title{
UK-South Asian patients' experiences of and satisfaction toward receiving information about biologics in rheumatoid arthritis
}

This article was published in the following Dove Press journal:

Patient Preference and Adherence

Kanta Kumar, ${ }^{1,2}$ Sabrina

R Raizada, ${ }^{3}$ Christian D

Mallen, ${ }^{4}$ Rebecca J Stack ${ }^{5}$

'Faculty of Biology, Medicine and Health, School of Health Sciences, The University of Manchester, Manchester, UK; ${ }^{2}$ Central Manchester University Hospitals Foundation Trust, Manchester, UK; ${ }^{3}$ The Royal Wolverhampton NHS Trust, Wolverhampton, UK; ${ }^{4}$ Arthritis Research UK Primary Care Centre, Primary Care Sciences, Keele University, Staffordshire, UK; ${ }^{5}$ School of Social Sciences, Nottingham Trent University, Nottingham, UK

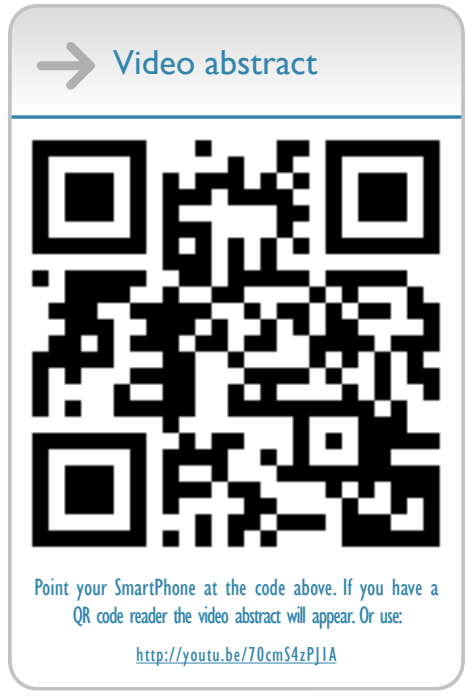

Correspondence: Kanta Kumar Faculty of Biology, Medicine and Health, School of Health Sciences, The University of Manchester, Oxford Road, Manchester MI3 9PL, UK Email kanta.kumar@manchester.ac.uk
Background: Rheumatoid arthritis (RA) causes painful joint inflammation and is incurable, but treatments control RA. Drug regimens are complex, and patients often do not take their medication as expected. Poor medication adherence can lead to poorly controlled disease and worse patient outcomes. Biologics treatments are expensive and require full engagement from patients. We have previously shown that patients from Black ethnic minority backgrounds do not fully engage into treatment plan. This study explored the patients' experiences in and satisfaction toward receiving information about biologics and future support preferences in South Asian patients with RA.

Methods: Twenty South Asian patients with RA from Royal Wolverhampton Hospitals NHS Trust and Central Manchester University Hospitals NHS Foundation Trust participated in individual semistructured interviews. Interviews were transcribed and data were analyzed by using thematic analysis approach.

Results: Four overarching themes describe the patients' experience in and satisfaction toward receiving information on biologics: 1) current provision of information regarding the "biologics journey" and understanding of RA: in this theme, non-English-speaking patients expressed heightened anxiety about stepping up to biologics; 2) experience and perceptions of biologics: many patients were positive about the biologic experience; however, there were patient-perceived delays in getting on to the biologics; 3 ) factors influencing willingness to try biologics: in this theme, a number of factors were identified including seeking advice from doctors abroad; and 4) recommendations on the desired information to fully understand the use of biologics: some patients valued group discussions, while others suggested receiving RA and biologic information through a video interaction.

Conclusion: This novel study provides insight into South Asian RA patients' experiences in and satisfaction toward receiving information about biologics. South Asian patients with RA reported a range of perceptions about biologics and support preferences, many of which may not be shared with the non-South Asian population.

Keywords: rheumatoid arthritis, biologic treatment, patient perceptions, illness beliefs, ethnicity

\section{Introduction}

Rheumatoid arthritis (RA) is a chronic inflammatory disease characterized by synovitis and associated joint destruction. ${ }^{1}$ RA causes progressive decline in functional ability and limits participation in social and work-related activities. ${ }^{2}$ RA is also associated with an increased risk of cardiovascular diseases (CVDs), ${ }^{3}$ which are worse among South Asian patients ${ }^{4}$ making inflammation control even more important in this patient group. While curative therapies are not currently available, early treatments with 
disease-modifying antirheumatic drugs (DMARDs) and biological agents improve joint function, ${ }^{1}$ work productivity, ${ }^{5}$ and health-related quality of life. ${ }^{6}$ Despite these treatments, many patients fail to achieve optimal disease control; this is in part due to nonadherence. ${ }^{7}$ Nonadherence has been reported to be a particular problem in South Asian patients with RA. ${ }^{8}$

Our previous work has shown concerns about treatments and poor medication adherence to be correlated with illness perceptions and cultural beliefs. Moreover, it is known that patients from South Asian backgrounds might discontinue RA treatments sooner compared with non-South Asian patients. ${ }^{9}$ It is only recently found that the provision of appropriate rheumatology care services for minority ethnic groups has begun to be substantively explored..$^{8,10-12}$ As the needs of particular minority ethnic groups become clearer, questions relating to the nature and scope of rheumatology care services have moved to the forefront of the minority ethnic group health care agenda. Since treating people with RA often involves using expensive therapies, it is surprising that there is only limited research available which considers the information needs of South Asian patients with RA taking biologics. The European League Against Rheumatism (EULAR) task force recommends guidelines for patient education in inflammatory arthritis. ${ }^{13}$ These recommendations clearly direct health professionals to move toward delivering tailored information and to meet the needs of individuals. However, tailored information for patients from ethnic backgrounds is limited in rheumatology. This study contributes to addressing this for one of the UK's largest minority ethnic populations. This study aims at exploring the perceptions of South Asians with RA in two prominent geographic areas on their experiences in and satisfaction toward receiving information about biologics and future support preferences.

\section{Materials and methods}

Ethical approval for this study was granted by the North West Greater Manchester South Research Ethics Committee (reference $17 / \mathrm{NW} / 0135)$, and all patients gave written informed consent.

The term "South Asian" here applies to people originating from the Indian subcontinent. Patients originating from the South Asian continent with a clinician-reported RA diagnosis were invited to participate in individual face-to-face and telephone interviews.

Patients were purposely sampled from secondary care rheumatology outpatient biologic clinics, giving a broad spectrum of age, disease duration, and gender. Two recruitment sites were used: Central Manchester University Hospitals
National Health Service (NHS) Foundation Trust and the Royal Wolverhampton NHS Trust. Patients were approached by the researcher (KK). Patient information sheet was provided outlining the aim of the study with those who had expressed an interest in taking part. A convenient time was organized with 20 patients for individual interviews. A topic guide was developed for the interview based on discussion with patient research partners and the study team (Figure 1). This was used to facilitate discussion in the individual interviews. These followed an iterative process, with new concepts emerging during data analysis being explored in subsequent interviews. A pro forma was designed to capture demographic data (Table 1).

Interviews were conducted at two hospitals, and eight patients were interviewed over the telephone for their convenience. The interviews were undertaken by KK who is fluent in English, Hindi, and Punjabi languages. Nine patients chose to be interviewed in Punjabi. Recruitment and interviewing continued until data saturation had been reached.

All interviews were digitally audiotaped, with interviews lasting up to 1 hour. Interviews were transcribed verbatim by an independent transcribing company. The transcripts in Punjabi were transcribed into English directly from audiotapes. A sample of transcriptions was independently checked for emerging themes by KK, RS, CM, and SR. ${ }^{14}$ Data were analyzed using thematic approach, ${ }^{15}$ in which initial analytical summaries of the interviews were coded line by line; the coding framework was discussed by the researchers, and coding categories that lacked concordance were absorbed back into the coding framework. The initial codes were then grouped into the most frequently occurring categories. Thematic analysis identified four themes that allowed the exploration of patients' experience about biologics and their expectations toward information, and future suggestions were also discussed.

\section{Results}

Twenty patients with RA participated in the study with an age range of 42-67 years. Disease duration ranged from 2 to 19 years. Of the 20 patients, three were male (refer Table 1 for complete demographic details).

\section{Current provision of information regarding biologics journey and understanding of RA}

Patients rated the quality of information delivered by health professional if they viewed that there was an opportunity to improve the quality of life (Q1; Table 2). The non-English-speaking patients expressed heightened 


\section{Experience of RA and DMARDs}

What is your perception of having RA?

How many different medicines have you taken?

What happened after you took these medicines?

\section{Experience of biologics}

Tell me about your experience of taking biologics?

How was the information given?

What information were you given by your nurse?

What information were you given by your doctor?

What information did you seek out yourself?

What information did other people give you about treatment?

Was the way we gave information about biologics appealing to you at that stage?

In what ways did information about your medicines influence your decision to taking biologics?

Did information ever make you want to stop taking your medicines, if so please describe what happened?

What thoughts were going through your mind when we stepped you up to this treatment?

Did you feel it was explained properly?

Did you know why the step-up occurred?

\section{Views on future content for biologics}

If we were to develop further material for future patients what would you advise to be the key points and information in that?

What ways do you think we should give patients information about medicines? What ways do you most like to receive health-related information?

What are your thoughts on medicines information being given to you in a standardized audio manner/format? What could clinicians and GPs do to encourage patients to get a better understanding of these medicines?

Figure I Topic guide used to facilitate interview discussions.

Abbreviations: DMARDs, disease-modifying antirheumatic drugs; RA, rheumatoid arthritis.

Table I Demographic data of the patients interviewed

\begin{tabular}{|c|c|c|c|c|c|}
\hline Gender & $\begin{array}{l}\text { Level of } \\
\text { education }\end{array}$ & Age & $\begin{array}{l}\text { Employment } \\
\text { status }\end{array}$ & $\begin{array}{l}\text { Disease duration } \\
\text { (years) }\end{array}$ & Current medication \\
\hline Patient I (female) & Primary & 54 & Retired & 10 & DMARDs, steroids, and biologic agents \\
\hline Patient 2 (female) & University & 45 & Retired due to RA & 2 & DMARDs and biologic agents \\
\hline Patient 3 (female) & Secondary & 53 & Factory worker & 5 & DMARDs and biologic agents \\
\hline Patient 4 (female) & University & 51 & Pharmacy technician & 4 & DMARDs and biologic agents \\
\hline Patient 5 (female) & Secondary & 42 & Nursery teacher & 10 & DMARDs and biologic agents \\
\hline Patient 6 (female) & Primary & 59 & Homemaker & 12 & DMARDs and biologic agents \\
\hline Patient 7 (female) & Secondary & 60 & Retired due to RA & 19 & DMARDs and biologic agents \\
\hline Patient 8 (female) & Primary & 62 & Homemaker & 18 & DMARDs and biologic agents \\
\hline Patient 9 (female) & Secondary & 55 & Factory worker & 9 & DMARDs and biologic agents \\
\hline Patient 10 (female) & Primary & 67 & Retired due to RA & 7 & DMARDs and biologic agents \\
\hline Patient II (male) & Secondary & 50 & Manager & 6 & DMARDs and biologic agents \\
\hline Patient I2 (male) & Secondary & 52 & Security officer & 9 & DMARDs, steroids, and biologic agents \\
\hline Patient I 3 (female) & University & 51 & Lawyer & 7 & DMARDs, steroids, and biologic agents \\
\hline Patient I4 (female) & Primary & 53 & Homemaker & 12 & DMARDs, steroids, and biologic agents \\
\hline Patient I5 (female) & Secondary & 64 & Retired due to RA & 14 & DMARDs, steroids, and biologic agents \\
\hline Patient I6 (female) & Secondary & 52 & Manager & 5 & DMARDs and biologic agents \\
\hline Patient I7 (male) & Secondary & 49 & Factory worker & 19 & DMARDs, steroids, and biologic agents \\
\hline Patient I8 (female) & University & 47 & Manager & 5 & DMARDs and biologic agents \\
\hline Patient 19 (female) & Secondary & 54 & Teacher assistance & 8 & DMARDs and biologic agents \\
\hline Patient 20 (female) & Primary & 51 & Factory worker & 7 & DMARDs and biologic agents \\
\hline
\end{tabular}

Abbreviations: DMARDs, disease-modifying antirheumatic drugs; RA, rheumatoid arthritis. 
Table 2 Quotes $(\mathrm{Q})$ relating to current provision of information regarding biologics journey and understanding of rheumatoid arthritis

QI: I had suffered so much and when they suggested this new medication [Enbrel] I just jumped to the chance of getting better than I was. My doctor told me all about how this medication was really helping many patients to get better so I thought why not give it a go. [male Englishspeaking patient]

Q2: When I look back at how many they have tried I didn't think anything would work and the side effects of these were worse than the others. [female non-English-speaking patient]

Q3: Well it takes three months to take effect you think right after three months something will happen then you discover about six months down the line nothing is happening then you change to another then another. When you go back to the rheumatology department they just try another one you do wonder if they know what they know what they are doing. [female English-speaking patient]

Q4: I have been through so many treatments I must say that I still don't understand why some of the treatments are not working ... if I am honest I don't understand the arthritis they say it's different in my knees than my hands. [female non-English-speaking patient]

Notes: The interviews were undertaken by KK, who is fluent in English, Hindi, and Punjabi languages. All interviews were digitally audiotaped. Interviews were transcribed verbatim by an independent transcribing company.

anxieties of stepping up of treatment and lack of familiarity with the process of switching to biologics (Q2; Table 2). There was some apprehension about health professional's explanation of biologics; patients often measured this by their experience using DMARDs and the length of time it took to commence biologics (Q3; Table 2). This notion was found in both English-speaking and non-English-speaking patients. Many patients expressed a lack of understanding of $\mathrm{RA}$; this appeared largely among the non-English-speaking patients (Q4; Table 2). Example quote Q1:

I had suffered so much and when they suggested this new medication [Enbrel] I just jumped to the chance of getting better than I was. My doctor told me all about how this medication was really helping many patients to get better so I thought why not give it a go. [male English-speaking patient]

\section{Experiences and perceptions on biologics}

There were a mixture of experiences in and perceptions on the use of biologics. Many patients viewed the biologics to be transforming their life (Q5; Table 3), which motivated them to engage with treatment plan (Q6; Table 3). Patients felt that the biologics were working better to control symptoms than DMARDs (Q7; Table 3). The perceptions on DMARDs and the poor efficacy of DMARDs to control symptoms masked the positive aspects of biologics for some patients (Q8; Table 3). For example, in some patients where there was a prolonged use of DMARDs (Q9; Table 3) and with many patients who questioned why the treatment was not available in the early stages of their disease (Q10; Table 3). Some viewed this time framework as "delay" or "time wasting" (Q11; Table 3). These perceptions led some patients to disengage with treatment plan and have less faith in health care professionals in controlling their RA (Q12; Table 3). Furthermore, the lack of doubt was displayed in the way that doctors in the Western countries managed their medicines for some; this was linked to their preference for doctors in India (Q13; Table 3). The notion of biologics being delayed in treatment journey was expressed by both English-speaking and non-English-speaking patients.

Table 3 Quotes $(\mathrm{Q})$ relating to experiences and perceptions of biologics
Q5: I was so much pain free and thought this was fantastic. I had my life back. [female English-speaking patient]
Q6: [...] To look at me now there is nothing wrong with me I am just a normal person. I can do everything in the day you know. [female English- speaking patient]
Q7: I do feel better with this drip now than the other medicines I tried. [female Punjabi-speaking patient]
Q8: I have tried so many things now I just don't know whether this new drug will work. I just keep thinking the others didn't so it is matter of waiting to see how well this will control the pain. [male Punjabi-speaking patient]
Q9: I don't [know] why I wasn't given these a lot sooner why wait so long I know they were saying my markers were low but my life was really disrupted by it all. [female English-speaking patient]
QI0: I do really think this all should [have] come sooner. I just couldn't understand why I had to wait three years. [male Punjabi-speaking patient] QII: If you ask me this has been all waste of time. I wasn't responding to any medications so why was there a long delay? [female Punjabi- speaking patient]
Q I2: It is very frustrating because I don't think the rheumatologist really understand what we as patients go through. After a while you just give up with them. [female English-speaking patient]
Q13: Well I was thinking if the first medicines didn't work that's why they are trying another one which I thought would. I had to go to India and speak to my doctor over there who told me these medicines would make me worse. [female Hindi-speaking patient]

Notes: The interviews were undertaken by KK, who is fluent in English, Hindi, and Punjabi languages. All interviews were digitally audiotaped. Interviews were transcribed verbatim by an independent transcribing company. Nine patients chose to be interviewed in Punjabi. The transcripts in Punjabi were translated and transcribed into English directly from the audiotapes. 
Example quote Q5: I was so much pain free and thought this was fantastic. I had my life back. [female Englishspeaking patient]

\section{Factors influencing willingness to try biologics}

The influencing factors varied and were part of decision making when considering biologics. However, it tended not to lead to a systematic search for scientific explanations, but rather to a reflection on personal experience and the experiences of family/relatives. Lay sources of information were frequently cited as a major influence on behavior. Patients expressed the need for better symptom control, and in some cases, patients anticipated biologics to cure the condition (Q14; Table 4). Given the degree of severity of their RA, some patients felt desperate to try new therapy (Q15; Table 4). Higher expectations were found to be influenced by information from sources such as clinicians, family members, and consultation with overseas doctors or by accessing the Internet. Some patients were satisfied with the explanation offered for stepping up therapy (Q16; Table 4); however, this was typically still validated with a family member for reassurance (Q17; Table 4). Furthermore, patients had explored the Internet and social media to get a general view on biologics. Many had used rheumatology charity sources for reassurances such as Arthritis Research UK (Q18; Table 4), but were generally less familiar with National Rheumatoid Arthritis Society (Q19; Table 4). It is clear from this fact that some patients may not be familiar with different sources of information. Arguably, this could reduce their chances of self-management.

The non-English-speaking patients who knew little about the biologics and appeared to not want information from the UK doctors and nurses sought advice from doctors abroad (Q20; Table 4). The information on patient self-management and the advice from overseas doctors may be more satisfactory for patients; however, it may not be evidence-based or in the best interest of preventing the long-term consequence of RA. Non-English-speaking patients' discussions with doctors abroad particularly took place in India. Often these doctors were found to be family members. The need for these discussions was based on the fact that they felt they knew very little about biologics and feared the side effect profile (Q21; Table 4). In some of the non-English-speaking patients, the validation process from doctors abroad and family members helped them to decide whether biologics should or should not to be taken (Q22; Table 4). There were a few patients who decided to avoid biologics mainly due to the side effect profile and were

Table 4 Quotes $(\mathrm{Q})$ relating to factors influencing willingness to try biologics

Q 14: Every time they tried a different medication I kept on thinking right this one will cure me. However, that didn't happen you know. but now I have accepted that this will not be cured it doesn't matter how hard they try. [female English-speaking patient]

Q I5: I was just thinking I want my career back and for me to being [be] a mum again. I was really lonely with other medications because they didn't do anything for me I felt lifeless but with this it was really great. I got back to work. [female English-speaking patient]

Q16: I had enough information plus I had looked up things myself. [male English-speaking patient]

Q I7: If it wasn't for my family members I wouldn't have been able to make a decision on this medication. [female English-speaking patient]

Q I8: I did look at Arthritis Research UK for more information. That I found useful but I hadn't heard of National Rheumatoid Arthritis Society. [male English-speaking patient]

Q19: I must say I haven't heard of National Rheumatoid Arthritis Society. I used social media to hear patient stories on tocilizumab. [female English-speaking patient]

Q20: I couldn't really understand why I was now going to take the injections. It did worry me. Yes, so, I did ask the doctor in India also my son said "mum don't take it until we ask the doctors over there" so when he told me that this was very serious medicines, I didn't feel it was for me. [female non-English-speaking patient]

Q2I: I am concerned about the side effects but what I can do? I have no other choice. The doctors in India said the look if you can't function then you have to take it. My children also said mum you have to have it that's the only way now to get better. [female non-English-speaking patient]

Q22: I will not take the medicine from here (UK) I am only taking the remedies given to me by the doctor in India. [female non-English-speaking patient]

Q23: I did look up at some herbal stuff and my husband wanted me to try them first. But soon I realised these were not going to do anything for me. The swelling was there I wasn't getting any better. [female non-English-speaking patient]

Q24: I did try the ayurvedic medicines. This did help at the start. I thought the swelling had gone down but this only lasted for a short time. [male English-speaking patient]

Q25: There are so many side effects to this [Humira] I don't think it is safe to take them. [female non-English-speaking patient]

Q26: I talked to my doctor in India and he gives the advice. [female non-English-speaking]

Notes: The interviews were undertaken by KK, who is fluent in English, Hindi, and Punjabi languages. All interviews were digitally audiotaped. Interviews were transcribed verbatim by an independent transcribing company. 
taking herbal remedies suggested by the doctors abroad and/ or by family members (Q23; Table 4). Both English-speaking and non-English-speaking patients had tried herbal remedies at some point in their journey. However, patients who did not achieve full symptom control reverted to biologics and did stop taking the herbal remedies (Q24 and Q25; Table 4). A few of the non-English-speaking patients did not view the use of biologics to be safe and desired to continue with the advice offered by doctors in India (Q26; Table 4). This might be related to the fact that the patients had more trust in the doctors abroad and felt comfortable with the advice given.

Example quote Q14: Every time they tried a different medication I kept on thinking right this one will cure me but that didn't happen you know but now I have accepted that this will not be cured it doesn't matter how hard they try. [female English-speaking patient]

\section{Recommendations on the desired information needed to fully understand the use of biologics}

Patients made recommendations for the future provision of information for biologics. There was a considerable variation in patients' understanding of the use of biologics and about RA in general (Q27; Table 5). Knowing information about their RA and the fluctuant nature of their disease influenced the way in which some patients managed their expectation of treatment plans (Q28; Table 5). While some patients were

Table 5 Quotes $(\mathrm{Q})$ relating to recommendations on the desired information to fully understand the use of biologics

Q27: There are lot of us who still don't understand what RA is. If the other medications [referring to DMARDs] didn't work then what to say these would? [female non-English-speaking patient]

Q28: I have to go with the flow really. Some days are good and some are not. RA does this so I have to take a day at a time really that's all you can do. [female English-speaking patient]

Q29: While you are trying so many medicines, you start thinking will I ever get better. You need that hope and motivation really. [female Englishspeaking patient]

Q30: Group discussions would be good to hear what others have found being on these medications. Sharing that experience would really help us because you do start to feel as if you are the only one suffering with this disease. [female English-speaking patient]

Q3I: If I had a something like a video to watch then my thoughts on my treatments would be different. You can should developed something like where you can see the person talking on this topic. [female non-English-speaking patient]

Q32: When I go out and start talking to my friends I hear more than I have in the department. You start feeling very lonely with this condition it will never go away so I think it would help to have something that we can view and listen to and feel more comfortable and think look I am not the only one with it. [female English-speaking patient]

Q33: I think it is really important to say that it is treatable I know we can't cure it so it can be controlled - that you are not alone in this - err and it is important to say it is continued treatable condition that the journey goes on. You need to say having the treatments gives you better life and how we can weigh up the side effects with benefits. In terms of the disease you don't have to suffer, I think there is great deal of perception it is seen as old person disease so you have to take that thought away. [female English-speaking patient]

Q34: In a lot of the Asian women there is the concept that it is what is it they start believing that they have to suffer. But in fact they can do something about it. Oldness is has changed now. If we want medicines to work we have to be part of that plan. So we have to get involved. [female English-speaking patient]

Q35: I think it should start from what this problem is about where it comes from, why it is better one day and not others, how we can help ourselves like one hand there is medication but what else can be done. [female non-English-speaking patient]

Q36: If I am honest one thing I couldn't understand why etanercept took so long to mention. I think if the doctors know these medications are coming in line why not explain this plan to patients. I think that would help having the feelings of non-hope. It is luck sometimes of which ones will work but the action plan should be discussed. [male patient English-speaking]

Q37: It is good to know that future research is still going on because that gives us hope you are trying for us and that would help us to get involved. [female non-English-speaking patient]

Q38: When I was first diagnosed I thought this could be cured but as time went on that didn't happen so you need to tell people what treatment does so that we can manage those expectations. [female English-speaking patient]

Q39: It is no good telling me after two years that there are still treatment options why don't you discuss everything at the start so that I can see what you are working with otherwise, my perception is rheumatologist don't know what they are doing. [female English-speaking patient] Q40: My GP is good I know that he took his time at the beginning but he gets all the letters from here and he reads them every time I go he will ask me how I am getting on. So they are important. [female English-speaking patient]

Q4I: The hospitals are busy so therefore I do hope that because my bloods at the GP practice and get my prescriptions from there. I do hope that I can get consistency with the GP let's see. I think better networking and better IT system between the two settings would enable us to work with both GP and hospital side. [male English-speaking patient]

Q42: I hardly see my consultant maybe every 12 months it would be nice to discuss things with GP between this time periods. [female Englishspeaking patient]

Notes: The interviews were undertaken by KK, who is fluent in English, Hindi, and Punjabi languages. All interviews were digitally audiotaped. Interviews were transcribed verbatim by an independent transcribing company. 
equipped with the relevant information and advice needed to make decisions, others lacked motivation and appropriate support to engage with RA management and treatment plan admitting, resulting in poor medication adherence and prolonged symptom control (Q29; Table 5). Some patients valued group discussions and suggested sharing good and negative experiences about biologics (Q30; Table 5), endorsing this to help reduce anxieties. Many of the patients suggested receiving $\mathrm{RA}$ and biologic information through a video interaction (Q31; Table 5) to help reduce the feelings of fear and isolation during their illness, thereby minimizing the burden of RA (Q32; Table 5). The video interaction may prove particularly beneficial for those with language barriers who may have inability to understand leaflets. Patients mentioned that having clips discussing the experiences of side effects, risk-benefit weighting, and emotional issues would all be highly valued (Q33; Table 5). This was suggested to be most useful for Asian women who might link RA to cultural barriers and view RA to be old persons' disease (Q34; Table 5). Most commented on the usefulness of having information in sections; for example, patients suggested an explanation on what is RA using pictorial diagrams, and they further proposed to know more on how and why RA causes fluctuant patterns (Q35; Table 5). Some suggested that they would value listening to how treatments work to control symptoms. Patients expressed to know more on why there is a delay in trying biologics in their RA journey (Q36; Table 5). Better information on the governing body on biologics such as the National Institute for Health and Care Excellence may help reduce the frustration regarding delays in trying biologics. Many suggested knowing the positives of the research taking place in RA and future goals (Q37; Table 5). There was a mention of expectations of treatments being too high with many suggesting that the initial DMARDs would control/ cure symptoms (Q38; Table 5). If structured information on RA was presented within early stages, then this would help patients manage their expectations and recognize the different levels of treatment plan (Q39; Table 5). Transparency in receiving information between secondary and primary care health care professionals was also noted to be paramount (Q40; Table 5). The synergy between rheumatology community and the general practitioners could play an active role in disseminating information on RA treatments (Q41 and Q42; Table 5) since many patients experienced a lack of contact with their hospital doctor and felt that GPs could add value to their experience.

Example quote Q27: There are lot of us who still don't understand what RA is and if the other medications [referring to DMARDs] didn't work then what to say these would? [female non-English-speaking patient]

\section{Discussion}

To our knowledge, this is the first qualitative study to have explored the perspectives of South Asians taking biologics and their satisfaction with information regarding biologics. In this study, we have identified interweaving factors that appeared to influence the experience of biologics. Many patients found biologics to be effective and were pleased with the results they produced despite their concerns of the long-term side effects. However, patients felt that there was a low level of satisfaction of biologic information. The impact of this appeared to be more on non-English-speaking patients. Indeed, this study has highlighted various issues and considerations that appear to have particular salience for South Asian patients with RA. The majority of South Asian patients had been living in the UK for most of their lives; the cultural and social practices of their countries of origin continued strongly to influence their decision for taking biologics. Family was the most essential source of support for South Asians in terms of both providing emotional support and assisting patients with decisions regarding biologics. For patients with RA from South Asian community, their condition is probably best understood within the context of their families or health professionals from their own background. If UK health care recommendations are not consistent with the belief systems of families and others, it is likely that those recommendations will not be followed. Moreover, this could lead South Asian patients to be at an increased risk of failing treatment target paradigm. ${ }^{16}$

An overriding concern expressed in all patients was the lack of available information relating to RA and the stages of treatment plan. The patients expressed a keen desire for this information to be made available mainly via video and/ or through interactive group discussion. The lack of available information is resulting in ignorance and fear of conventional medicines leading many patients to seek advice on traditional medicines from doctors or other practitioners abroad. Informed education, therefore, is clearly essential to influence how people manage RA and their expectations toward a treatment plan. Some of the themes reported in our study were similar to other work exploring biologic information. ${ }^{17}$ For example, in those studies, patients were also found to be concerned about long-term effects of biologics. ${ }^{18}$ Moreover, the patients were similar in their views about having received biologics at a later stage in their journey. ${ }^{19}$ However, the new and fundamental pattern in our study was the constant validation of biologics with family members and seeking 
advice from doctors abroad. The lack of trust in the UK rheumatology clinicians could be detrimental to the long-term disease management and medication adherence. In addition, we found that lack of knowledge of RA was often found to impact on treatment decisions and, importantly, patients' view on the opportunity to be cured rather than controlled. This was not cited in other studies investigating non-South Asian patients' perceptions on biologics. ${ }^{20}$

As has been noted throughout, many of the findings reported in this study were similar to the findings of our previous research where we reported lack of knowledge of RA and influence of illness perceptions resulting in poor medication adherence. ${ }^{8}$ Our work also reflects a wider problem that health professionals face in providing effective information on RA and treatment to minority ethnic populations. ${ }^{8}$ This could also be problematic for rheumatology charities who aim at supporting patients with RA. However, patient suggestions made in this study for future support could be an opportunity for both health professionals and rheumatology charities to work closely with South Asian patients. Furthermore, recommendations made by the patients in this study align with EULAR guidelines on patient education that suggest reflecting on approaches to educating patients with RA. ${ }^{13}$

Lessons can also be learnt from other chronic disease areas, such as CVDs, where researchers designed innovative interventions to specifically address ethnic minority. For example, "Khush Dil" (happy heart) ${ }^{21}$ is a project aimed at delivering education in a culturally acceptable manner. A separate culturally adapted lay-led self-management programme for Bangladeshi adults with chronic disease found an improvement in self-care. ${ }^{22}$ Moreover, a systematic review of educational interventions among the ethnic minority groups strongly recommended the involvement of patients and urged education interventions to include culturally sensitive components for better clinical outcomes. ${ }^{23}$ Health education that concords with people's "lay treatment and disease views" is more likely to lead to changes in behavior than that which seems to contradict such models. The video interaction containing patient-centered information may encourage patients to take control of RA and decisions about treatment.

Our study had a number of limitations. We recruited patients with a range of disease duration; it could be that patients in whom there was a longer use of DMARDs could have viewed the RA and biologics experience differently than those who started biologics sooner. This required an in-depth comparison between the two groups. There were a limited number of male patients in the study; therefore, their perceptions on RA and biologics could have differed from the perspectives of female patients.

\section{Conclusion}

These novel data focusing on South Asian patients' experiences in using biologics and their satisfaction toward information received suggest that they have a range of experiences from being on biologics and a range of recommendations to address the issues they experience. The issues identified as specific to South Asians suggest that some of these experiences, level of satisfaction with information, and support preferences may not be shared with non-South Asians. Therefore, there is a realistic possibility that many South Asian patients with RA are not being served by relevant and appropriate information that could have an impact on treatment target paradigm. The findings may help health professionals to understand and build strategies that might assist patients to map their journey with RA and treatments more effectively.

\section{Acknowledgments}

We thank all of the patients for taking part in the study and staff at the Royal Wolverhampton Hospitals NHS Trust and Central Manchester University Hospitals NHS Foundation Trust. This study was funded by the Manchester Institute for Collaborative Research on Ageing. CM is funded by the National Institute for Health Research (NIHR) Collaborations for Leadership in Applied Health Research and Care (CLAHRC), West Midlands, the NIHR SPCR, and a NIHR Research Professorship in General Practice (NIHR-RP2014-04-026). The views and opinions expressed herein are those of the authors and do not necessarily reflect those of the NIHR (UK).

\section{Author contributions}

All authors contributed toward data analysis, drafting and critically revising the paper, gave final approval of the version to be published, and agree to be accountable for all aspects of the work.

\section{Disclosure}

The authors report no conflicts of interest in this work.

\section{References}

1. Emery P. Treatment of rheumatoid arthritis. BMJ. 2006;332:152-155.

2. National Audit Office Report. Services for people with rheumatoid arthritis; 2009. Available from: http://www.nao.org.uk/publications/0809/ rheumatoid_arthritis.aspx. Accessed May 5, 2017.

3. Kitas GD, Gabriel SE. Cardiovascular disease in rheumatoid arthritis: state of the art and future perspectives. Ann Rheum Dis. 2011;70:8-14. 
4. Grover S, Sinha RP, Singh U, Tewari S, Aggarwal A, Misra R. Subclinical atherosclerosis in rheumatoid arthritis in India. J Rheumatol. 2006;33: 244-247.

5. Young A, Dixey J, Kulinskaya E, et al. Which patients stop working because of rheumatoid arthritis? Results of five years' follow up in 732 patients from the Early RA Study (ERAS). Ann Rheum Dis. 2002;61: $335-340$

6. Symmons DP, Prior P, Scott DL, Brown R, Hawkins CF. Factors influencing mortality in rheumatoid arthritis. J Chronic Dis. 1986;39: 137-145.

7. Pasma A, van't Spijker A, Hazes JM, Busschbach JJ, Luime JJ. Factors associated with adherence to pharmaceutical treatment for rheumatoid arthritis patients: a systematic review. Semin Arthritis Rheum. 2013; 43:18-28.

8. Kumar K, Raza K, Nightingale P, et al. Determinants of adherence to disease modifying anti-rheumatic drugs in White British and South Asian patients with rheumatoid arthritis: a cross sectional study. $B M C$ Musculoskelet Disord. 2015;16:396.

9. Helliwell PS, Ibranhim G. Ethnic differences in responses to disease modifying drugs. Rheumatology (Oxford). 2003;42:1197-1201.

10. Kumar K, John H, Gordhan C, Situnayake D, Raza K, Bacon PA. Breaking communication barriers for RA patients of south Asian origin: the use of a bilingual educational audio $\mathrm{CD}$ and linguistically appropriate peer support and education. Musculoskeletal Care. 2011;9: 11-18.

11. Kumar K, Daley E, Khattak F, Buckley CD, Raza K. The influence of ethnicity on the extent of, and reasons underlying, delay in general practitioner consultation in patients with RA. Rheumatology (Oxford). 2010;49:1005-1012.

12. Kumar K, Raza K, Gill P, Greenfield S. The impact of using musculoskeletal ultrasound imaging and other influencing factors on medication adherence in patients with rheumatoid arthritis: a qualitative study. Patient Prefer Adherence. 2016;10:1091-1100.

13. Zangi HA, Ndosi M, Adams J, et al. EULAR recommendations for patient education for people with inflammatory arthritis. Ann Rheum Dis. 2015;74:954-962.
14. Greenhalgh T, Taylor R. Papers that go beyond numbers (qualitative research). BMJ. 1997;315:740-743.

15. Dixon-Woods M, Agarwal S, Jones D, Young B, Sutton A. Patients' perceptions of treatment with anti-TNF therapy for rheumatoid arthritis: a qualitative study. Rheumatology. 2004;43:1034-1038.

16. Smolen JS, Landewe R, Breedveld FC, et al. EULAR recommendations for the management of rheumatoid arthritis with synthetic and biological disease-modifying antirheumatic drugs: 2013 update. Ann Rheum Dis. 2014;73:492-509.

17. Marshall NJ, Wilson G, Lapworth K, Kay LJ. Patients' perceptions of treatment with anti-TNF therapy for rheumatoid arthritis: a qualitative study. Rheumatology (Oxford). 2004;43:1034-1038.

18. Barton JL. Patient preferences and satisfaction in the treatment of rheumatoid arthritis with biologic therapy. Patient Prefer Adherence. 2009;3:335-344.

19. Sanderson T, Calnan M, Morris M, Richards P, Hewlett S. The impact of patient-perceived restricted access to anti-TNF therapy for rheumatoid arthritis: a qualitative study. Musculoskeletal Care. 2009;7:194-209.

20. Arkell P, Ryan S, Brownfield A, Cadwgan A, Packham J. Patient experiences, attitudes and expectations towards receiving information about anti-TNF medication - "It could give me two heads and I'd still try it!". BMC Musculoskelet Disord. 2013;14:165.

21. Mathews G, Alexander J, Rahemtulla T, Bhopal R. Impact of a cardiovascular risk control project for South Asians (Khush Dil) on motivation, behaviour, obesity, blood pressure and lipids. J Public Health (Oxf). 2007;29:388-397.

22. Griffiths C, Motlib J, Azad A, et al. Randomised controlled trial of a lay-led self-management programme for Bangladeshi patients with chronic disease. Br J Gen Pract. 2005;55:831-837.

23. Netto G, Bhopal R, Lederle N, Khatoon J, Jackson A. How can health promotion interventions be adapted for minority ethnic communities? Five principles for guiding the development of behavioural interventions. Health Promot Int. 2010;25:248-257.
Patient Preference and Adherence

\section{Publish your work in this journal}

Patient Preference and Adherence is an international, peer-reviewed, open access journal that focuses on the growing importance of patient preference and adherence throughout the therapeutic continuum. Patient satisfaction, acceptability, quality of life, compliance, persistence and their role in developing new therapeutic modalities and compounds to optimize

\section{Dovepress}

clinical outcomes for existing disease states are major areas of interest for the journal. This journal has been accepted for indexing on PubMed Central. The manuscript management system is completely online and includes a very quick and fair peer-review system, which is all easy to use. Visit http://www. dovepress.com/testimonials.php to read real quotes from published authors. 